\title{
Supporting Information \\ A Micropatterned Multifunctional Carbohydrate Display by an Orthogonal Self-Assembling Strategy
}

\author{
Hajime Sato, ${ }^{1}$ Yoshiko Miura, ${ }^{* 2,4}$ Nagahiro Saito, ${ }^{1}$ \\ Kazukiyo Kobayashi, ${ }^{2}$ and Osamu Takai 1,3
}

\begin{abstract}
1Department of Materials, Physics and Energy Engineering, Graduate School of Engineering, Nagoya University, Furo־cho, Chikusa-ku, Nagoya 464-8603, Japan.

2Department of Molecular Design and Engineering, Graduate School of Engineering, Nagoya University, Furo-cho, Chikusa-ku, Nagoya 464-8603, Japan.

${ }^{3}$ EcoTopia Science Institute, Nagoya University, Furo-cho, Chikusa-ku, Nagoya 464-8603, Japan.

${ }^{4}$ School of Materials Science, Japan Advanced Institute of Science and Technology, 1-1 Asahidai, Nomi, Ishikawa 923-1292, Japan
\end{abstract}

\section{Supporting Information Data}

\section{A. Materials.}

The following reagents were used as received: octadecyltrimethoxysilane (ODS) and 3-aminopropyltrimethoxysilane (APS) (Gelest, Inc., Morrisville, PA); fluorescein isothiocyanate (FITC)-labeled Ricinus communis agglutinin $120\left(\mathrm{RCA}_{120}\right)$ (Honen Co. Ltd., Tokyo, Japan); basic fibroblast growth factor (bFGF) (BioCarta, Inc., San Diego, CA); tetramethylrhodamine 5-isothiocyanate (5-TRITC, G isomer, Molecular Probes, Eugene, OR); heparin from Hog intestine (TCI Co. Ltd., Tokyo, Japan); Dulbecco's modified Eagle medium (DMEM) (Gibco, Grand Island, NY); penicillin, streptomycin, fetal calf serum (FCS), and bovine insulin (Sigma Chemicals, St. Louis, MO).

bFGF was labeled with TRITC.1,2 A bFGF solution ( $3 \mathrm{~mL}, 0.01 \mathrm{mg} / \mathrm{mL}$ ) was prepared in $0.10 \mathrm{M}$ Borax buffer at $\mathrm{pH} 9.2$. TRITC $(0.1 \mathrm{mg})$ in $0.1 \mathrm{~mL}$ of DMSO was added to the protein solution, and the mixture was stirred at $4{ }^{\circ} \mathrm{C}$ for $6 \mathrm{~h}$. The solution was dialyzed in PBS for 3 days. The resulting TRITC-labeled bFGF was analyzed by fluorescence spectrometry.

PVLA $\left(\mathrm{M}_{\mathrm{w}}=1.7 \times 10^{4}, \mathrm{M}_{\mathrm{w}} / \mathrm{M}_{\mathrm{n}}=2.4\right)$ was prepared according to our previous method and purified by dialysis using a cellulose tube with $\mathrm{M}_{\mathrm{w}}$ cut-off $=3,500$ (Nacalai Tesque, Kyoto, 
Japan) ${ }^{3}$

B. Measurements.

The thickness of the substrate was estimated using an ellipsometer (PZ2000, Philips, Eindhoven, Holland) equipped with a He-Ne laser of wavelength $632.8 \mathrm{~nm}$ operating at an incident angle of $70^{\circ}$. X-ray photoelectron spectroscopy (XPS) was performed with an X-probeTM 206 spectrometer (Surface Science Instruments, Mountain View, CA) equipped with a monochromated AlK $\alpha$ source of $200 \mathrm{~W}$ (1000 mm spot) at a collection angle of $55^{\circ}$ from the normal. Scanning electron microscopy (SEM) was performed with a JSM-6330F microscope (JEOL Ltd, Japan). The micropatterns of FITC-RCA120 and TRITC-bFGF were visualized with a Zeiss Axiovert $200 \mathrm{M}$ laser confocal microscope (Carl Zeiss Inc., Gottingen, Germany) equipped with an external argon laser (for excitation at $488 \mathrm{~nm}$ ). The fluorescence staining was imaged with a GenePix 4000B scanner (Union, $\mathrm{CA})$.

\section{Micropatterned ODS/APS Substrates.}

Method:

According to our previously reported method, ${ }^{4}$ ODS-SAMs were prepared on cleaned silicon substrates or glass substrates by CVD (chemical vapor deposition). The ODS-SAM was micropatterned by irradiating the surface with VUV (vacuum ultraviolet) light for 30 min with an excimer lamp (UER20-172V, $\lambda=172 \mathrm{~nm}$ and $10 \mathrm{~mW} / \mathrm{cm}^{2}$, Ushio Inc., Tokyo, Japan) through a photomask of $50 \times 125 \mu \mathrm{m}$ spots (for characterizations) or $200 \times 500 \mu \mathrm{m}$ spots (for coculture). The micropatterned ODS-SAM substrate was then immersed in a toluene solution of $1 \mathrm{vol} \%$ APS at $60{ }^{\circ} \mathrm{C}$ under $\mathrm{N}_{2}$, and sonicated in toluene, ethanol, and Milli-Q water to remove excess APS. Silicon substrates were used for XPS, ellipsometry, and SEM, and glass substrates were used for fluorescence microscopy and optical microscopy.

\section{Results:}

The micropatterned ODS/APS substrates in Figure 2 was prepared by the following consecutive treatments:5 (1) chemical vapor deposition (CVD) of ODS, (2) photoirradiation of the ODS-SAM with VUV $(172 \mathrm{~nm})$ through a photomask, and (3) the subsequent treatment of the photoirradiated substrate with APS. The XPS spectra of each ODS and APS region of the micropatterned ODS/APS substrates on silicon are shown respectively in Figure 3(a-1) and $(\mathrm{b}-1)$. A C1s peak at $286 \mathrm{eV}$ on the ODS-SAM substrate was decreased on the irradiated regions (the data not shown) and recovered by the subsequent treatment of the ODS-SAM with APS. A large N1s signal around $403 \mathrm{eV}$ 
of amino group also appeared on APS region (the data not shown) and the peak intensity ratio of $\mathrm{C} / \mathrm{N}$ was approximately $24: 1$. Each thickness of ODS region and APS region was estimated by elllipsometry to be $15 \AA$ and $8 \AA$, respectively. Figure S-1 illustrates the micropatterned ODS/APS substrate observed by SEM. A bright and dark binary pattern appeared along ODS and APS region, respectively. Well-organized micropatterning of hydrophobic and cationic characters could be attained via the photolithography of ODS-SAM using VUV and the successive APS-SAM formation.

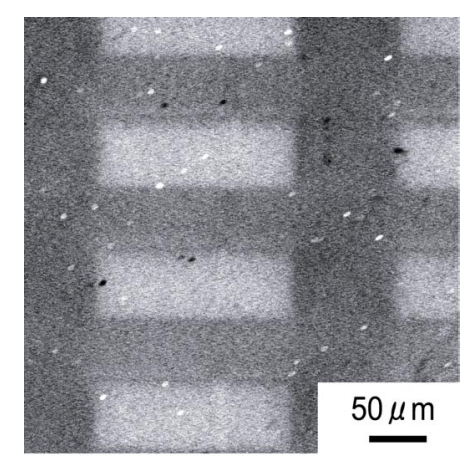

Figure S-1. A SEM image of micropatterned ODS/ APS substrate on silicon.

D. Molecular Recognition by $\mathrm{RCA}_{120}$ and bFGF.

Method:

ODS/APS-SAM on glass substrates were successively immersed in each heparin $(0.05$ $\mathrm{mg} / \mathrm{ml}, 5 \mathrm{~h})$ and PVLA $(0.5 \mathrm{mg} / \mathrm{ml}, 3 \mathrm{~h})$ solution in this order and treated with FITC-labeled RCA R20 $(0.1 \mathrm{mg} / \mathrm{ml}, 1 \mathrm{~h})$ and TRITC-labeled bFGF $(0.01 \mathrm{mg} / \mathrm{ml}, 1 \mathrm{~h})$, separately.

Result:

The self-assembly of heparin occurred only on the APS region, but the adsorption of PVLA was not enough selective on the ODS region. Then, ODS/APS substrates on glass were successively immersed first in each heparin and then in PVLA solution in this order, and the resulting (PVLA-ODS)/(Heparin-APS) substrates were treated separately with FITC-labeled RCA 120 and TRITC-labeled bFGF. As shown in Figure S-2(a), the green fluorescence of FITC-labeled RCA 120 was detected on the PVLA-ODS region, with an S/N ratio of 3.1. The red fluorescence of TRITC-labeled bFGF was detected on the heparin-APS region, with an S/N ratio of 3.6 in Figure $\mathrm{S}-2(\mathrm{~b})$. 
(a)
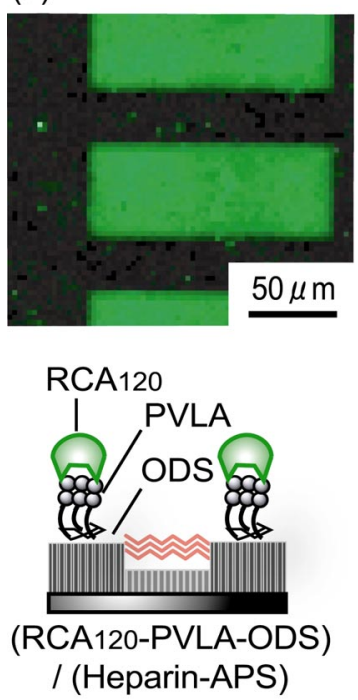

(b)
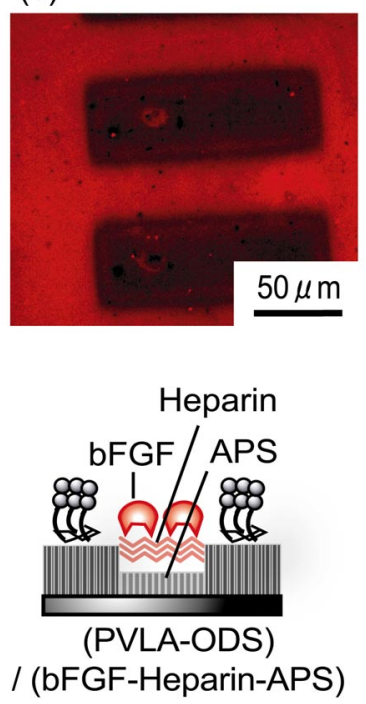

Figure S-2. Fluorescence images of (PVLA-ODS)/(Heparin-APS) substrates treated with (a) FITC-RCA 120 and (b) TRITC-bFGF.

E. References

1. Dubey, I.; Pratviel, G.; Meunier, B. Bioconjugate Chem. 1998, 9, 627.

2. Gajraj, A.; Ofoli, R. Y. Langmuir 2000, 16, 8085.

3. Kobayashi, K.; Sumitomo, H.; Ina, Y. Polym. J. 1985, 17, 567.

4. Miura, Y.; Sato H.; Ikeda, T.; Sugimura, H.; Takai, O.; Kobayashi, K. Biomacromolecules 2004, 5, 1708.

5. (a) Sugimura, H.; Hanji, T.; Takai, O.; Masuda, T.; Misawa, H. Electrochim. Acta 2001, 47, 103. (b) Saito, N.; Wu, Y.; Hayashi, K.; Sugimura, H.; Takai, O. J. Phys. Chem. B. 2003, 107, 664. (c) Hayashi, K.; Saito, N.; Sugimura, H.; Takai, O.; Nakagiri, N. Langmuir 2002, 18, 7469. 